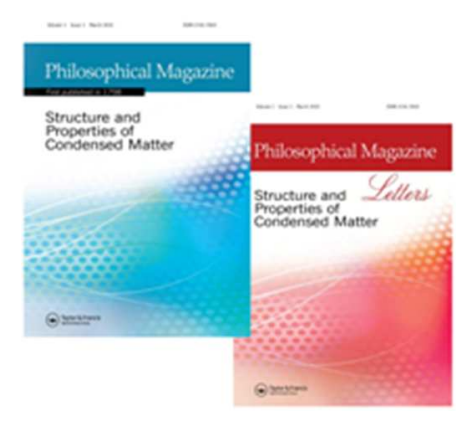

\title{
Temperature dependence of formation and shrinkage of hollow shells in hemispherical Ag/Pd nanoparticles
}

\begin{tabular}{|r|l|}
\hline Journal: & Philosophical Magazine \& Philosophical Magazine Letters \\
\hline Manuscript ID: & TPHM-12-Jan-0007.R1 \\
\hline Journal Selection: & Philosophical Magazine \\
\hline Date Submitted by the Author: & n/a \\
\hline Keywords: & $\begin{array}{l}\text { Gisf of Authors: } \\
\text { Physics } \\
\text { Cserhati, Csaba; University of Debrecen, Department of Solid State Physics }\end{array}$ \\
\hline Keywords (user supplied): & hollow nanostructures, stability, Kirkendall effect \\
\hline &
\end{tabular}


Temperature dependence of formation and shrinkage of hollow shells in hemispherical Ag/Pd nanoparticles

Györgyi Glodán ${ }^{\mathrm{a}}$ Csaba Cserháti ${ }^{\mathrm{a}}$ and Dezső L. Beke ${ }^{\mathrm{a}}$ *

${ }^{\mathrm{a}}$ Department of Solid State Physics, University of Debrecen, 4010 Debrecen, P.O.Box. 2, Hungary

*Corresponding author. E-mail: dbeke@delfin.unideb.hu 


\title{
Temperature dependence of formation and shrinkage of hollow shells in hemispherical Ag/Pd nanoparticles
}

\author{
Györgyi Glodán $^{1)}$, Csaba Cserháti ${ }^{1)}$ and Dezső L. Beke ${ }^{1)}$ \\ ${ }^{1)}$ Department of Solid State Physics, University of Debrecen, 4010 Debrecen, P.O.Box. 2, \\ Hungary
}

It is shown that both the growth and shrinkage of hollow shells in $\mathrm{Ag} / \mathrm{Pd}$ hemispherical core-shell nano structures took place at the same temperature. The crossover time, $t_{\mathrm{cr}}$, between these regimes is shifted to smaller values with increasing temperatures. This result confirms that the growth and the shrinkage regimes are controlled by the faster as well as the slower diffusion coefficients $\left(D_{\mathrm{Ag}}\right.$ as well as $\left.\mathrm{D}_{\mathrm{Pd}}\right)$, respectively. The pore radius, confirming recent theoretical predictions, linearly depends on the initial particle radius and the slope of this straight line increases with the average composition of the faster component.

Keywords: hollow nanostructures; stability; interdiffusion; Kirkendall effect

\section{Introduction}

The synthesis of hollow nanostructures (nanospheres, nanotubes) have promising properties for new types of possible applications such as recoverable catalysts, drug delivers, photonic devices, nano-chemical reactors etc. [1-13]. In most of the cases the formation of nanospheres or nanotubes was based on the solid state diffusion and/or reaction between two parent materials and the Kirkendall effect was applied to explain the formation of holes in the core, containing the fast diffusing element.

Indeed it was shown theoretically that the formation stage of pores inside a core/shell (A/B) nanosphere was controlled by the diffusion coefficient of the fast diffusion component $[3,4,9,12]$; a resultant vacancy flow, $J_{v}$, oriented to the A core, arises because $J_{A}>J_{B}\left(D_{A}>D_{B}\right.$, $\mathrm{D}_{\mathrm{i}}$ are the diffusion coefficients). Although the details of the nucleation and growth of the $\operatorname{void(s)~can~be~complicated~due~to~different~additional~effects~(stress~development~}[4,10,14]$, non-steady state vacancy distribution [12], etc.) it is generally accepted that the overall growth of the hole is controlled by $D_{A}$ and $t_{g} \sim R_{0}{ }^{2} / D_{A}$ was obtained for the time to complete the shell formation $[4,11]$ ( $R_{0}$ is the initial particle radius).

On the other hand the above nanoshell structure should be unstable due to the GibbsThomson effect [6,7], creating an outward vacancy flux leading to shrinkage. In binary systems the so called inverse Kirkendall effect should also influence the shrinkage $[7,8]$. Indeed it was obtained in [7] that the shrinking time (during which the radius of the hole decreases to the $\mathrm{R}_{\mathrm{ho}} / e$ value, where $e$ is the base of the natural logarithm and $\mathrm{R}_{\mathrm{ho}}$ is the initial hole radius) can be given as

$$
t_{s h r} \sim(k T / \gamma \Omega)\left(R_{f}^{3} / D_{A}\right)\left[\left(1-c_{A}\right) D_{A} / D_{B}+c_{A}\right] .
$$

$\mathrm{R}_{\mathrm{f}}$ is the final particle radius after collapse, $\mathrm{k}$ and $\mathrm{T}$ have their usual meaning, $\mathrm{c}_{\mathrm{A}}$ is the atomic fraction of $\mathrm{A}, \gamma$ and $\Omega$ denote the surface energy and the atomic volume, respectively. If $D_{A} / D_{B} \gg>1, t_{s h r} \sim R_{f}^{3} / D_{B}$. In recent theoretical papers $[8,9,11,12,15,16]$ it is shown that, in 
binary systems with wide mutual solubility range and for relatively not too large $D_{A} / D_{B}(\cong 10)$ ratios, first there is a relatively fast growing stage which is followed by a slower shrinkage process at the same temperature. This behaviour was experimentally illustrated in $\mathrm{Ag} / \mathrm{Au}$ system [10], where an $\mathrm{Ag}-50 \% \mathrm{Au}$ solid solution has been formed in the shell and the shrinkage took place at the same temperature. All earlier experimental investigations were carried out in systems, where the shell was a reaction product and the shrinkage process was observed at higher temperatures than at which they were formed (see e.g. [13] where Ni and $\mathrm{Cu}$ oxide nanoshells were produced by oxidation of pure metallic particles).

In a very recent theoretical paper [11] the growth and shrinkage process in one run was investigated in both spherical and cylindrical geometry. It was found that the maximal pore radius, corresponding to the crossover time, $t_{c r}$, between the two regimes, depended (almost linearly) on the initial particles radius, $R_{o}$, and on the average concentration. Unfortunately the effect of the annealing temperature was not simulated.

In this communication we report experimental results on the temperature dependence of the porosity formation and shrinkage in $\mathrm{Ag} / \mathrm{Pd}$ hemisphere sells.

\section{Experimental}

Separated hemisphere beads of Ag were produced as described in [10]. The average initial radius, of the $\mathrm{Ag}$ islands was about $20 \mathrm{~nm}$ as well as $10 \mathrm{~nm}$ for two different sets of samples. Thin Pd film (of about 10-20nm) was evaporated on the top of ensembles of $\mathrm{Ag}$ beads. The initial average radii of the $\mathrm{Ag} / \mathrm{Pd}$ hemispherical particles, $\mathrm{R}_{\mathrm{o}}$, was about 40 as well as $27 \mathrm{~nm}$ (Set 1 and Set 2, respectively) with a dispersion of about 35\% (Fig. 1).

The specimens were annealed at $703 \mathrm{~K}, 723 \mathrm{~K}$ and $743 \mathrm{~K}$ for $10,20,30,60,120$ and 180 minutes in dynamic reducing gas flow $(5 \% \mathrm{H}+95 \%$ Ar mixture $)$. After heat treatments the beads were removed from the sapphire substrate by tearing off the dried film formed by dropping collodion (solution of nitrocellulose in alcohol) solution onto the surface. The dried film was placed with the top side onto a TEM grid covered with amorphous carbon layer. After the dissolution of the carrier (collodion) layer the bottom side of the beads was investigated by JEOL $2000 \mathrm{FX}-\mathrm{II}$ TEM/EDX system at $200 \mathrm{keV}$ on plan view.

The area of the holes and beads were measured by standard image processing method: the edges of the beads and pores were detected at several points. The points were then connected with straight lines and the area of this polygon had been measured in $\mathrm{nm}^{2}$.

Fig. 2 shows examples of the hollow islands obtained at $743 \mathrm{~K}, 723 \mathrm{~K}$ and $703 \mathrm{~K}$ after 20 minutes. The formed pores have circular or polygonal shape. Usually less than tree pores (but mostly only one pore) were evolved during the heat treatments. Note, that an opposite phenomena was observed in the Ag-Au system; many small pores were observed at the lowest annealing temperature, similarly as predicted by computer simulations [7] and observed also during Co sulphide shell formation [4].

Fig. 3 shows the ratio of the area of the pore (a) and particle (A) versus the annealing time at different temperatures for Set 1 . This kind of presentation is beneficial, because the area ratio clearly mimics the volume or radius ratio and because the initial size of our beads showed a size distribution $\left(\mathrm{a} / \mathrm{A} \sim \mathrm{R}_{\mathrm{p}} / \mathrm{R}_{\mathrm{e}}\right.$, where and $\mathrm{R}_{\mathrm{p}}$ and $\mathrm{R}_{\mathrm{o}}$ are the pore radius and the external radius of the shell). The functions in Fig. 3 are similar to the same plots obtained the in $\mathrm{Ag} / \mathrm{Au}$ system [1] and it is in accordance with predictions of theoretical papers $[9,11,12]$ : the envelope curves have the maximum at the crossover time, $t_{\mathrm{cr}}$, between the growth and shrinkage regimes. However, in contrast to [10], where we could not draw a definite conclusion on the temperature dependence of $t_{c r}$, the temperature dependence of $t_{c r}$ can be 
clearly seen in Fig. 3: the position of the maximum is shifted to longer times with decreasing temperature. At $743 \mathrm{~K}$ the maximum should be at about 10 minutes, i.e. the formation of pores was too fast to be observed (see the dashed line in Fig. 3).

In both of our experiments (in this one and also in [10]) a distribution of beads was present. Thus it was possible to plot the pore radius, $\mathrm{R}_{\mathrm{p}}$, as the function of the external radius, $\mathrm{R}_{\mathrm{e}}$, belonging to the maxima of the relative pore area versus time functions. Linear relations were obtained in [10] for $a$ versus $A$ (or $\mathrm{R}_{\mathrm{p}}$ versus $\mathrm{R}_{\mathrm{e}}$ ) functions with similar slopes for both temperatures investigated. In Fig. 4, in order to make easier the comparison with recent theoretical predictions [11], the radius of the pore at $t_{\mathrm{cr}}$, versus the actual (external) radius of the particles, are plotted directly in this system. In spite of the high experimental scatter of the points, the functions at a fixed annealing temperature can be fitted with a linear function. Increasing the temperature the slope of the linear function also increases. (In Fig. 4, for the shake of clarity, the points belonging to the intermediate temperature, $723 \mathrm{~K}$, are not shown, but they lie between the two straight lines).

Having two sample sets, with different initial average radii, $\mathrm{R}_{\mathrm{o}}$, we can also investigate whether the slopes of the $R_{p}$ versus $R_{e}$ functions are the same in these two ensembles or not. Fig. 5 shows these two functions at $743 \mathrm{~K}$. The points can be fitted by straight lines, with different slopes: the difference in slopes is about a factor of two.

\section{Discussion and conclusions}

It can be seen in Fig. 3 that the time corresponding to the maxima of the curves, $t_{c r}$, shifts to smaller values with increasing temperature. According to theoretical estimations for larger $t_{\mathrm{g}} / \mathrm{t}_{\text {shr }}$ ratio the crossover time should be shorter, i.e. $t_{\mathrm{cr}} \sim t_{\text {shr }} / \mathrm{t}_{\mathrm{g}} \sim\left(\mathrm{R}_{\mathrm{f}}{ }_{\mathrm{f}} / \mathrm{R}_{\mathrm{o}}{ }^{2}\right) \mathrm{D}_{\mathrm{A}} / \mathrm{D}_{\mathrm{B}} \cong \mathrm{R}_{\mathrm{o}} \mathrm{D}_{\mathrm{A}} / \mathrm{D}_{\mathrm{B}}$ (due to conservation of matter $\left(R_{f} / R_{0}\right)^{3} \cong 1$, i.e. $R_{f} \cong R_{0}$.) Since $D_{A} \gg D_{B}$ and, as a general rule, the diffusion activation energy for the slower component is larger than the faster one $\left(\mathrm{Q}_{\mathrm{B}}>\mathrm{Q}_{\mathrm{A}}\right)$;

$$
\mathrm{t}_{\mathrm{cr}} \sim \exp \left[\left(\mathrm{Q}_{\mathrm{B}}-\mathrm{Q}_{\mathrm{A}}\right) / \mathrm{kT}\right] \text {, }
$$

if $R_{0}$ is constant. This indeed gives decreasing $t_{c r}$ values with increasing temperatures. Thus we can conclude that the temperature dependence of the crossover time observed is in accordance with the theoretical result that the growth and the shrinkage processes are controlled by the faster as well as slower diffusing components, respectively.

It follows from the previous arguments that if $\mathrm{D}_{\mathrm{A}}$ and $\mathrm{D}_{\mathrm{B}}$ differs considerably, (e.g. $D_{A}>D_{B}$ i.e. $D_{A} / D_{B}>10^{3}$ ) then the shrinkage process can be observed only at higher temperatures than the temperature at which their formation was observed. This seems to be the case for oxide nanoshells or nanotubes formed by oxidation of metallic particles $[13,17]$. If the $D_{A} / D_{B}$ ratio is not considerably larger than unity then, being the times $t_{\text {shr }}$ and $t_{g}$ close values, the two stages can be observed in one run, but the wide maximum on the $R_{p}$ versus $t$ plots does not have a well recognizable shift with the temperature. This was the case in the $\mathrm{Ag} / \mathrm{Au}$ system (see Fig. 2. in [10]). For intermediate values of $\mathrm{D}_{\mathrm{A}} / \mathrm{D}_{\mathrm{B}}$ the two stages can be still observable at the same temperature and the temperature dependence of the crossover time can also be observed. Let us see numerical values for the diffusion coefficients. According to data collection [18] $\mathrm{D}_{\text {AginAg }} / \mathrm{D}_{\text {AuinAg }} \cong 9$ while $\mathrm{D}_{\mathrm{AginAg}} / \mathrm{D}_{\text {PdinAg }} \cong 300$ at $740 \mathrm{~K}$. Obviously these ratios are composition dependent and e.g. $\mathrm{D}_{\mathrm{AginAu}} / \mathrm{D}_{\mathrm{AuinAu}} \cong 2$ (there are no experimental for $\mathrm{D}_{\text {AginPd }}$ ), but their average value of this ratio over the whole composition range is still should differ by about a factor of 5 . Thus one can conclude that indeed in the $\mathrm{Ag} / \mathrm{Au}$ system the two stages should be not far from each other, while in the $\mathrm{Ag} / \mathrm{Pd}$ system the growth and shrinkage 
times are different enough to allow the observation of the temperature dependence of the crossover time.

Theoretical calculations in [11] resulted in a linear dependence of the pore radius, $R_{p}$, at the crossover moment, as the function of the initial particle radius $\left(R_{0}\right)$. From our experimental data we were able to calculate the above pore radius as the function of the actual (external) particle radius, $\mathrm{R}_{\mathrm{e}}$. However, according to the conservation of volume, $\mathrm{R}_{\mathrm{e}}{ }^{3}=\mathrm{R}_{\mathrm{o}}{ }^{3}+\mathrm{R}_{\mathrm{p}}{ }^{3}$, and following a similar approximation used also in [7], i.e. $\mathrm{R}_{\mathrm{o}}-\mathrm{R}_{\mathrm{p}}=\Delta \mathrm{R}<\mathrm{R}_{\mathrm{o}}$, one can write:

$$
\mathrm{R}_{\mathrm{e}}{ }^{3} \cong 2 \mathrm{R}_{\mathrm{o}}{ }^{3}\left\{1-\left(3 / 2\left(\Delta \mathrm{R} / \mathrm{R}_{\mathrm{o}}\right)\left[1-\Delta \mathrm{R} / \mathrm{R}_{\mathrm{o}}\right]\right\} \text {, i.e. } \mathrm{R}_{\mathrm{e}} \sim \mathrm{R}_{\mathrm{o}} .\right.
$$

Thus our results, shown in Fig. 4 , can be interpreted as the experimental confirmation of the linear relation between $R_{p}$ and $R_{o}$ (see also Fig. 3 in [11]). Moreover, we can also conclude from our results that the slope of this straight line has definite temperature dependence: the slope increases with increasing temperature.

The results shown in Fig. 5 are also interesting. At first sight it seems to be surprising why the slopes of the straight lines, fitted to the two ensembles with different average initial radii, differ. The smaller slope can be interpreted as follows. In [11] it was shown that the slope of the $R_{p}$ versus $R_{o}$ functions depends on the average composition of the faster component, $\mathrm{c}_{\mathrm{A}}$, as well. It was obtained (Fig. 4 in [11]) that the slope was smaller for lower average value of $c_{A}$. In our experiments the average composition of the formed AgPd solid solutions could be different for the two groups with different average initial radii. Indeed, according to the Energy Dispersive X-ray analysis (EDX) in TEM, the Ag content was about 65 at $\%$ as well as 54at\% in the hollow hemispherical shells in the sets with larger and smaller average radius, respectively. Thus the silver content should be less for particles with smaller initial radius and thus, in accordance with the calculations [11], the slope for this group should be smaller too.

\section{Acknowledgments}

This work was supported by Grant No. CK 80126 of the Hungarian Scientific Research Found and and by the TAMOP 4.2.1./B-09/1/KONV-2010-007 project, which is co-financed by the European Union and European Social Fund.

\section{References}

[1] Y. Sun, B. Mayers, Y. Xia, Nano Lett. 2 (2002) p. 481

[2] Y. Sun, B. Mayers, Y. Xia, Adv. Mater. 25 (2003) p. 641

[3] Y.Yin, R.M. Rioux, C.K. Erdonmez, S. Hughes, G,A, Somorjai, A.P. Alivisatos, Science 304 (2004) p. 711

[4] Y. Yin, C.K. Erdonmez, A. Cabot, S. Hughes, A.P. Alivisatos, Adv. Func. Mater. 16 (2006) p. 1389

[5] H.J. Fan, M. Knez, R. Scholtz, D. Hesse, K. Nirelsch. M. Zacharias, U. Gössele, Nano Lett. 7 (2007) p. 993

[6] K. N. Tu, U. Gössele, Appl. Phys. Lett. 86 (2005) p. 093111

[7] A.M. Gusak. T.V Zaporozhets, K.N. Tu, U. Gössele, Phil. Mag. 85 (2005) p. 4445

[8] A.V. Evteev, A.V. Levchenko, I.V. Belova, G.E. Murch, Phil. Mag. 88 (2008) p. 1525

[9] A.M. Gusak, T.V. Zaporozhets, J. Phys. Condens. Matter. 21 (2009) p. 415303

[10] Gy. Glodán, Cs. Cserhati, I. Beszeda, D.L. Beke, Appl. Phys. Lett. 97 (2010) p. 113109

[11] O.M. Podolyan, T.V. Zaporozhets, Ukr. J. Phys. 56 (2011) p. 929 
[12] G.E. Murch, A.V. Evteev. E.V. Levtheko, I,V, Belova, Diffus. Fundam. 42 (2009) p. 1 [13] R. Nakamura, D. Tokozakura, J.-G.Lee, H. Mori, H. Nakajima, Acta Mater. 56 (2008) p. 5276

[14] J. Svoboda, F.D. Fisher, Acta Mater. 59 (2011) p. 61

[15] A.M. Gusak, K.N. Tu, Acta Mater. 57 (2009) p. 3367

[16] A.V. Evteev, A.V. Levchenko, I.V. Belova, G.E. Murch, J. of Nano Res. 7 (2009) p. 11

[17] R. Nakamura, G. Matsubayashi, H. Tsuchiya, S. Fujimoto, H. Nakajima, Acta Mater. 57 (2009) p. 5046

[18] H. Mehrer (editor): Diffusion in Solid Metals and Alloys, Landolt Börnstein, New Series III/26, Springer-Verlag, Berlin, 1990

Figure captions:

Fig. 1 Size distribution of the initial Ag/Pd hemispherical particles for the two sets of samples.

FIG 2: TEM picture of Ag-Pd hemispheres formed at a) $743 \mathrm{~K}$, b) $723 \mathrm{~K}$ and c) $703 \mathrm{~K}$ for 20 minutes annealing time

FIG 3: Relative area of the pores as the function of the annealing time at $703 \mathrm{~K}$ (square), $723 \mathrm{~K}$ (triangle) and $743 \mathrm{~K}$ (circle) for Set 1 . The experimental points were obtained by averaging over 10-30 particles.

FIG 4: Hole radius, $R_{p}$, at the crossover time as the function of the actual (external) radius, $R_{e}$, of the beads for $703 \mathrm{~K}$ (square) and $743 \mathrm{~K}$ (circle) (a, b, c are the slopes of the linear functions at 743,723 and $703 \mathrm{~K}$, respectively) for Set 1.

FIG 5: Hole radius, $R_{p}$, at the crossover time as the function of the actual (external) radius, $R_{e}$, at $743 \mathrm{~K}$ for the two sets of samples with different initial sizes ( $\mathrm{a}$, and $\mathrm{b}$ are the slopes of the linear functions, belonging to the bigger (circle) and smaller (triangle) average initial sizes, respectively). 
Size distribution of the initial Ag/Pd hemispherical particles for the two sets of samples. $120 \times 103 \mathrm{~mm}(300 \times 300 \mathrm{DPI})$ 


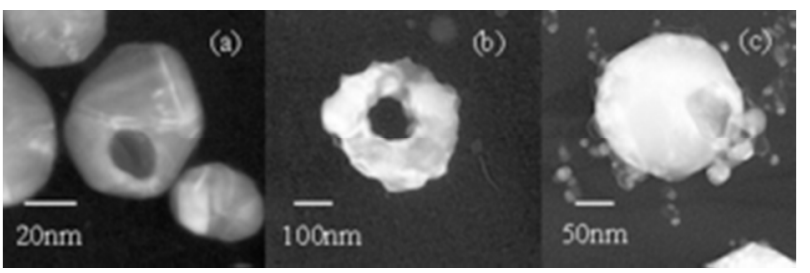

$25 \times 8 \mathrm{~mm}(300 \times 300 \mathrm{DPI})$ 


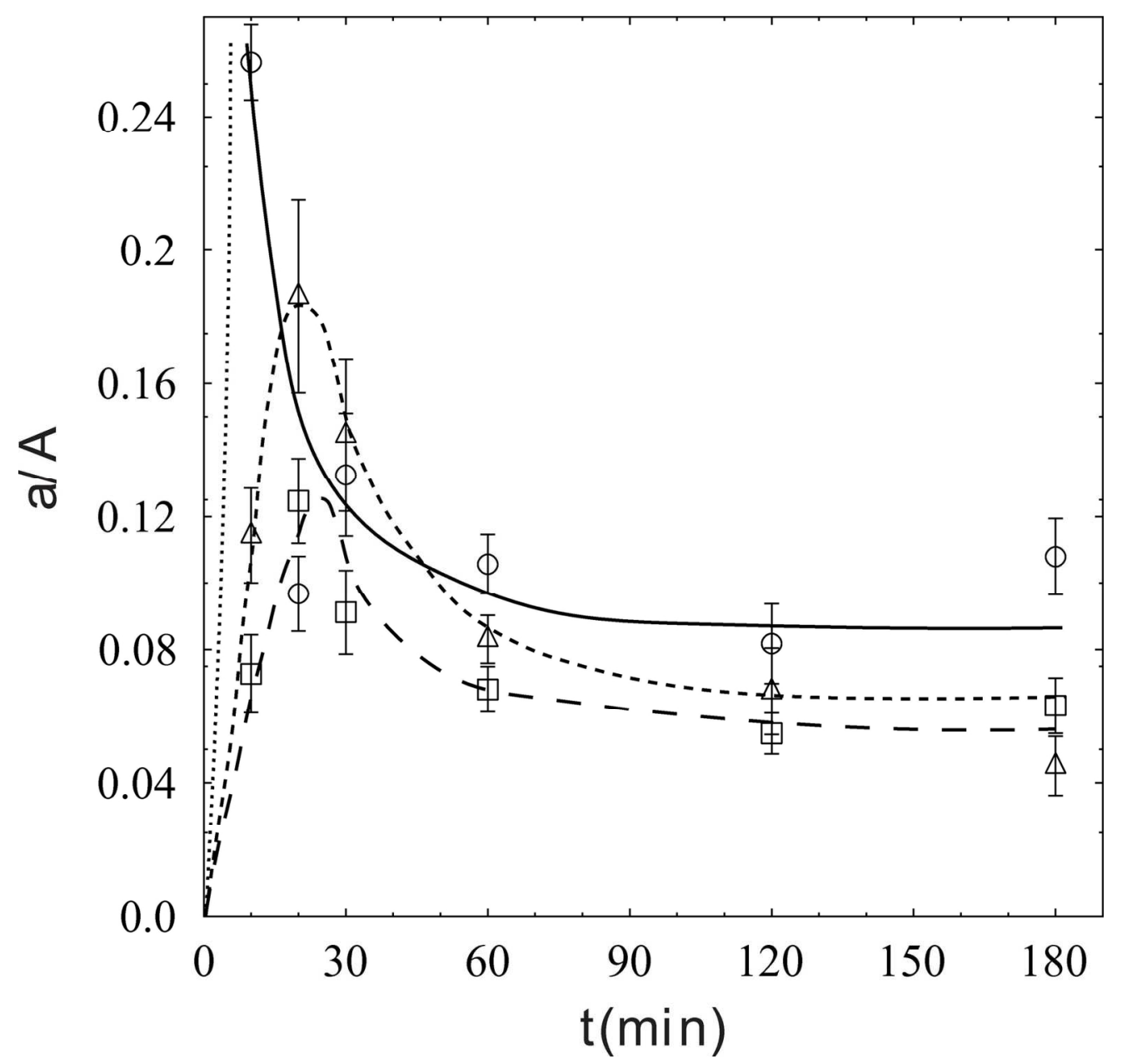

Relative area of the pores as the function of the annealing time at $703 \mathrm{~K}$ (square), $723 \mathrm{~K}$ (triangle) and $743 \mathrm{~K}$ (circle) for Set 1 . The experimental points were obtained by averaging over 10-30 particles. $145 \times 139 \mathrm{~mm}(300 \times 300 \mathrm{DPI})$ 
Hole radius, $\mathrm{Rp}$, at the crossover time as the function of the actual (external) radius, $\mathrm{Re}$, of the beads for $703 \mathrm{~K}$ (square) and $743 \mathrm{~K}$ (circle) ( $\mathrm{a}, \mathrm{b}, \mathrm{c}$ are the slopes of the linear functions at 743, 723 and $703 \mathrm{~K}$, respectively) for Set 1. $120 \times 103 \mathrm{~mm}(300 \times 300 \mathrm{DPI})$ 
Hole radius, $\mathrm{Rp}$, at the crossover time as the function of the actual (external) radius, $\mathrm{Re}$, at $743 \mathrm{~K}$ for the two sets of samples with different initial sizes ( $a$, and $b$ are the slopes of the linear functions, belonging to the bigger (circle) and smaller (triangle) average initial sizes, respectively). $120 \times 103 \mathrm{~mm}(300 \times 300$ DPI $)$ 